\title{
The impact of preoperative sarcopenia, defined based on psoas muscle area, on long-term outcomes of heart valve surgery
}

\author{
Homare Okamura, MD, PhD, ${ }^{\mathrm{a}, \mathrm{b}}$ Naoyuki Kimura, $\mathrm{MD}, \mathrm{PhD},{ }^{\mathrm{a}}$ Keisuke Tanno, $\mathrm{MD}, \mathrm{PhD},{ }^{\mathrm{c}}$ \\ Makiko Mieno, $\mathrm{PhD},{ }^{\mathrm{d}}$ Harunobu Matsumoto, $\mathrm{MD}, \mathrm{PhD},{ }^{\mathrm{a}}$ Atsushi Yamaguchi, $\mathrm{MD}, \mathrm{PhD},{ }^{\mathrm{a}}$ and \\ Hideo Adachi, MD, $\mathrm{PhD}^{\mathrm{b}}$
}

\section{ABSTRACT}

Objective: Sarcopenia, age-related loss of muscle mass, is an objective and comprehensive marker of frailty. We aimed to clarify the influence of sarcopenia on the outcomes after heart valve surgery.

Methods: We retrospectively reviewed 1119 patients who underwent valve surgery via median sternotomy at our institution from June 2009 to December 2013. Patients aged $<70$ years, urgent/emergent cases, and patients without preoperative computed tomography of the abdomen were excluded. The remaining 428 patients were included in this study. Psoas muscle area, a validated measure of sarcopenia, was measured on preoperative computed tomography. Sarcopenia was defined as the lowest sex-specific quartile in psoas muscle area. The mean follow-up period was 3.4 years.

Results: Overall in-hospital mortality did not differ between the sarcopenia and nonsarcopenia patient groups. However, the incidence of stroke and intra-aortic balloon pump/percutaneous cardiopulmonary support use was greater in the sarcopenia group than in the nonsarcopenia group. The patients with sarcopenia had significantly decreased long-term survival and decreased freedom from major adverse cardiac and cerebrovascular events. Multivariable analysis and inverse probability weighting revealed that sarcopenia was an independent predictor for decreased survival (hazard ratio, 2.22; 95\% confidence interval, 1.26-3.92; $P=.006)$.

Conclusions: Preoperative sarcopenia defined from the psoas muscle area was associated with long-term outcomes after valve surgery. Thus, the measurement of psoas muscle area can help facilitate more accurate risk scoring in elderly patients. (J Thorac Cardiovasc Surg 2019;157:1071-9)

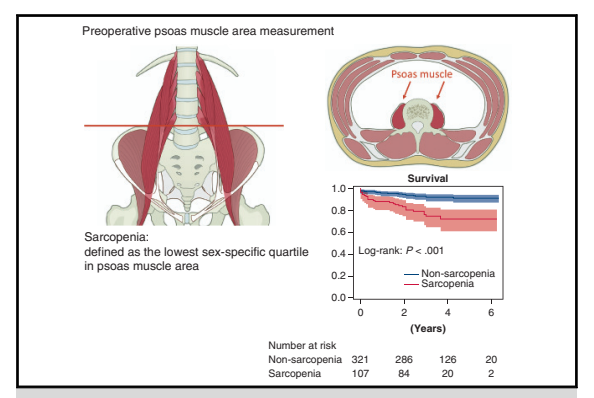

Sarcopenia has a negative impact on long-term outcomes of heart valve surgery.

\section{Central Message}

Preoperative assessment of sarcopenia by measuring the psoas muscle area on computed tomography is a simple method to identify frail patients and predict long-term outcomes after valve surgery.

\section{Perspective}

Sarcopenia is the age-related loss of muscle and has been recognized as a marker of frailty. However, the impact of sarcopenia on the outcomes after cardiac surgery remains unknown. The assessment of sarcopenia based on the psoas muscle area measured on preoperative computed tomography helps identify frail patients and predicts long-term outcomes after heart valve surgery.

See Commentary on page 1080.

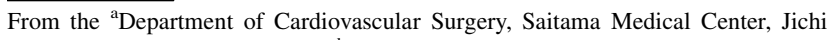
Medical University, Saitama; ${ }^{b}$ Department of Cardiovascular Surgery, Nerima Hikarigaoka Hospital, Tokyo; ${ }^{\mathrm{c} D e p a r t m e n t ~ o f ~ R a d i o l o g y, ~ S a i t a m a ~ M e d i c a l ~ C e n t e r, ~}$ Jichi Medical University, Saitama; and ${ }^{\mathrm{d}}$ Department of Medical Informatics, Center for Information, Jichi Medical University, Tochigi, Japan.

Source of funding: Grant-in-Aid for Young Scientists B (\#17K16598 to H.O.) from the Ministry of Education, Culture, Sports, Science and Technology, Japan.

Received for publication Dec 4, 2017; revisions received June 2, 2018; accepted for publication June 17, 2018; available ahead of print Aug 20, 2018.

Address for reprints: Homare Okamura, MD, PhD, Department of Cardiovascular Surgery, Saitama Medical Center, Jichi Medical University, 1-847, Amanuma-cho, Omiya-ku, Saitama-shi, Saitama, 330-8503, Japan (E-mail: homareokamura@ omiya.jichi.ac.jp).

$0022-5223 / \$ 36.00$

Copyright (c) 2018 by The American Association for Thoracic Surgery

https://doi.org/10.1016/j.jtcvs.2018.06.098
}

In the aging population, the decision-making regarding the operability of elderly and morbid patients has increasingly been an issue of contention. The heterogeneity of comorbidity and activity that exists in this patient population renders the decision-making process more challenging. As such,

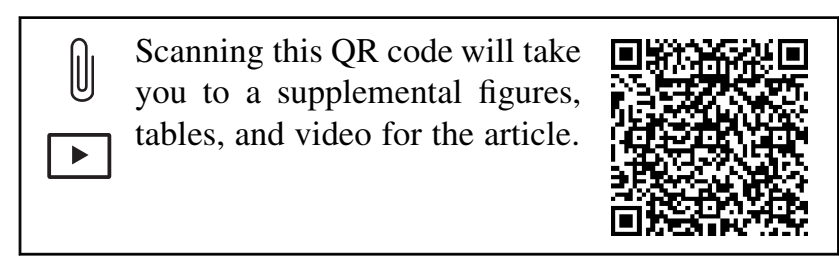




$$
\begin{aligned}
& \text { Abbreviations and Acronyms } \\
& \begin{aligned}
\mathrm{BMI} & =\text { body mass index } \\
\mathrm{CT} & =\text { computed tomography } \\
\mathrm{MACCE} & =\text { major adverse cardiac and } \\
& \text { cerebrovascular events } \\
\mathrm{PS} & =\text { propensity score }
\end{aligned}
\end{aligned}
$$

preoperatively identifying patients at high risk for mortality and morbidity has become more important. Furthermore, technological advances in cardiac surgery have led to various treatment options for each condition and have also made risk assessment more essential to provide optimal treatment to each patient.

Several risk models, such as the European System for Cardiac Operative Risk Evaluation II and the Society of Thoracic Surgeons score, have been used to stratify perioperative risk in cardiac surgery. ${ }^{1-3}$ However, these conventional risk models have traditionally focused more on the presence or absence of specific medical comorbidities and do not incorporate frailty, age-related systemic vulnerability, which is considered to have negative impacts on the outcomes. It is also unclear whether these risk-stratification systems can predict long-term survival after cardiac surgery.

Frailty is a geriatric syndrome reflecting impaired physiologic reserve and a decreased resistance to internal and external stressors across multiple physiologic systems. Both the aging process and some chronic diseases interact with each other and result in frailty. Within the aging population, frailty becomes increasingly prevalent and is associated with a considerable social and economic burden. ${ }^{4}$

Recently, frailty has been proposed as a means to help predict the outcomes after surgery., ${ }^{5,6}$ Afilalo and colleagues ${ }^{5}$ showed that slow gait speed is an independent predictor of operative mortality and major morbidity after cardiac surgery. However, most measures of frailty are based on a matrix of several physical function and mental tests, which makes frailty hard to define. In connection with frailty, sarcopenia is the age-related loss of muscle mass and function, which is closely related to poor physical performance. Not surprisingly, many of the adverse results of frailty stem from the loss of skeletal muscle mass. Sarcopenia is present in about $5 \%$ to $10 \%$ of individuals older than 65 years of age. ${ }^{7}$ Some investigators have reported that patients with sarcopenia had a greater likelihood of long-term mortality after gastrointestinal surgery. ${ }^{8,9}$ However, the impact of sarcopenia on the clinical outcomes after cardiac surgery still remains unclear. In this study, we aimed to assess whether preoperative sarcopenia can be used to predict the outcomes after heart valve surgery in elderly patients.

\section{PATIENTS AND METHODS \\ Patient Population and Study Design}

This was a retrospective study based on our institutional database. Between June 2009 and December 2013, a total of 1119 patients underwent heart valve surgery via median sternotomy at Saitama Medical Center, Jichi Medical University. We excluded patients $<70$ years old, urgent/emergent cases, and patients with missing preoperative computed tomography (CT) of the abdomen data used for the assessment for sarcopenia. Thus, 428 patients were included in this study (Figure 1).

First, we divided the patients into 2 groups, those with and those without sarcopenia, based on psoas muscle area measured with preoperative abdominal CT. Preoperative CT was performed within 3 months before surgery. Subsequently, perioperative patient characteristics, in-hospital outcomes, and long-term outcomes were compared between the patients with and those without sarcopenia.

Our study was approved by the institutional review board of Jichi Medical University (March 28, 2017, no. S16-014). Follow-up data including survival and complications were obtained via a patient chart review, questionnaires mailed to patients, and/or by telephone. The mean follow-up period was 3.4 years, and the follow-up rate was $100 \%$.

\section{Surgical Procedure}

Surgeries were performed via median sternotomy. Cardiopulmonary bypass was established with aortic-bicaval cannulation. Moderate hypothermia was applied, and antegrade/retrograde cold blood cardioplegia was administered intermittently. A left ventricular vent was inserted via the right upper pulmonary vein. Surgical procedures are shown in Table 1. Warfarin sodium was started on the day of surgery and continued after surgery so that the international normalized ratio of prothrombin time was maintained in accordance with the American College of Cardiology/American Heart Association guidelines. ${ }^{10}$ In patients with a tissue valve, warfarin sodium was discontinued 3 months after surgery, unless there was another indication for anticoagulation.

\section{Definition of Sarcopenia}

We measured psoas muscle area, a validated marker of sarcopenia, on the preoperative CT scan. Using the Ziostation (Ziosoft Inc, Tokyo, Japan), we measured the psoas muscle area at the level of the top of the iliac crest in all patients and averaged (Figure 2, A). A radiologist (K.T) measured

Heart valve surgery performed via median sternotomy at Saitama Medical Center, Jichi Medical University between June 2009 and December $2013(n=1119)$

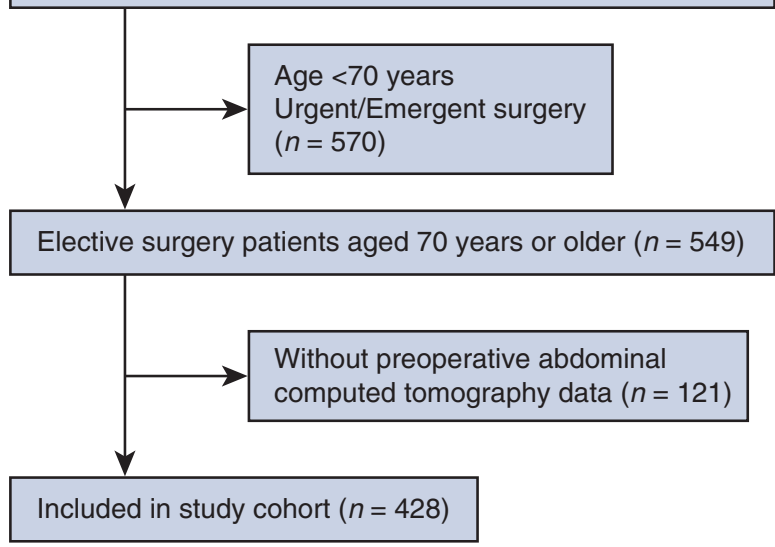

FIGURE 1. Patient flow diagram. 
TABLE 1. Preoperative patient characteristics per study group

\begin{tabular}{|c|c|c|c|c|}
\hline Variable & Total $(n=428)$ & Sarcopenia $(n=107)$ & Nonsarcopenia $(\mathbf{n}=321)$ & $P$ value \\
\hline Age, y & $76.3 \pm 4.4$ & $77.0 \pm 4.6$ & $76.0 \pm 4.3$ & .065 \\
\hline Sex, female & $222(51.9 \%)$ & $55(51.4 \%)$ & $167(52.0 \%)$ & .912 \\
\hline Body surface area, $\mathrm{m}^{2}$ & $1.49 \pm 0.17$ & $1.40 \pm 0.13$ & $1.52 \pm 0.16$ & $<.001$ \\
\hline Body mass index, $\mathrm{kg} / \mathrm{m}^{2}$ & $21.9 \pm 3.6$ & $19.6 \pm 2.8$ & $22.6 \pm 3.5$ & $<.001$ \\
\hline NYHA class $3 / 4$ & $160(37.4 \%)$ & $51(47.7 \%)$ & $109(34.0 \%)$ & .015 \\
\hline Hypertension & $302(70.6 \%)$ & $71(66.4 \%)$ & $231(72.0 \%)$ & .327 \\
\hline Diabetes & $95(22.2 \%)$ & $23(21.5 \%)$ & $72(22.4 \%)$ & .894 \\
\hline Ischemic heart disease & $97(22.7 \%)$ & $27(25.2 \%)$ & $70(21.8 \%)$ & .505 \\
\hline Atrial fibrillation & $134(31.3 \%)$ & $36(33.6 \%)$ & $98(30.5 \%)$ & .630 \\
\hline Cerebrovascular disease & $53(12.4 \%)$ & $14(13.1 \%)$ & $39(12.1 \%)$ & .865 \\
\hline Chronic kidney disease* & $47(11.0 \%)$ & $19(17.8 \%)$ & $28(8.7 \%)$ & .013 \\
\hline Hemodialysis & $17(4.0 \%)$ & $10(9.3 \%)$ & $7(2.2 \%)$ & .003 \\
\hline COPD & $12(2.8 \%)$ & $7(6.5 \%)$ & $5(1.6 \%)$ & .013 \\
\hline Peripheral artery disease & $25(5.8 \%)$ & $9(8.4 \%)$ & $16(5.0 \%)$ & .232 \\
\hline Previous cardiac surgery & $17(4.0 \%)$ & $5(4.7 \%)$ & $12(3.7 \%)$ & .775 \\
\hline LVEF \% & $60.8 \pm 12.5$ & $59.2 \pm 12.6$ & $61.3 \pm 12.4$ & .156 \\
\hline Serum hemoglobin, $\mathrm{g} / \mathrm{dL}$ & $12.1 \pm 1.7$ & $11.5 \pm 1.6$ & $12.2 \pm 1.7$ & $<.001$ \\
\hline Serum albumin, $\mathrm{g} / \mathrm{dL}$ & $4.0 \pm 0.5$ & $3.9 \pm 0.5$ & $4.1 \pm 0.4$ & $<.001$ \\
\hline Psoas muscle area, $\mathrm{mm}^{2}$ & $758 \pm 262$ & $530 \pm 143$ & $834 \pm 248$ & $<.001$ \\
\hline
\end{tabular}

Values for continuous variables are expressed as mean \pm standard deviation, and values for categorical variables are expressed as numbers (\%). NYHA, New York Heart Association; $C O P D$, chronic obstructive pulmonary disease; $L V E F$, left ventricular ejection fraction. *Serum creatinine $>1.5 \mathrm{mg} / \mathrm{dL}$.

the area by manually outlining the borders of both psoas muscles (Figure 2, $B$ ). In this study, sarcopenia was defined as the lowest sex-specific quartile in the average of both psoas muscle areas at preoperative CT scan, in accordance with what was done in previous studies. ${ }^{8,9,11-14}$

\section{Statistical Analysis}

The data in this study are expressed as mean \pm standard deviation for continuous variables and as frequency and percentage for categorical variables. Between-group comparison for categorical variables was performed by the Fisher exact test. The Student $t$ test or Mann-Whitney $U$ test was used for the comparison of the continuous variables as appropriate. Low cardiac output syndrome was defined as (1) requiring intra-aortic balloon pump/percutaneous cardiopulmonary support to be weaned from cardiopulmonary bypass and/or (2) the need for adrenaline or more than $5 \mu \mathrm{g} / \mathrm{kg} / \mathrm{min}$ dopamine or dobutamine, after correction of electrolytes and preload, in the intensive care unit. Long-term survival and freedom from major adverse cardiac and cerebrovascular events (MACCE) were estimated via the Kaplan-Meier method, and comparisons between 2 groups were performed with the log-rank test. MACCE was defined as acute myocardial infarction, angina, congestive heart failure, new cardiac arrhythmia, stroke, cardiovascular death, and/or cerebrovascular death. ${ }^{15}$

Forward stepwise Cox proportional hazards regression analysis was performed to evaluate the impact of preoperative and intraoperative data on survival and freedom from MACCE. Variables included age; sex; body surface area; body mass index (BMI) $<18.5 \mathrm{~kg} / \mathrm{m}^{2}$, BMI $>30.0 \mathrm{~kg} / \mathrm{m}^{2}$; New York Heart Association class 3/4; hypertension; diabetes; ischemic heart disease; atrial fibrillation; stroke; chronic kidney disease (serum creatinine $>1.5 \mathrm{mg} / \mathrm{dL}$ ); hemodialysis; chronic obstructive pulmonary disease; peripheral arterial disease; history of cardiac surgery; left ventricular ejection fraction; serum hemoglobin; serum albumin; and intraoperative data (type of surgical procedure).
Variables with $P<.20$ on univariate analysis were entered into a multivariable Cox proportional hazards regression analysis. To adjust for all major confounders between the sarcopenia and nonsarcopenia groups, the inverse probability weighting using propensity score (PS) analysis was performed. ${ }^{16}$ PS was constructed based on the aforementioned confounders, and each observation was weighted by its inverse probability of being in a certain group. PS-weighted Kaplan-Meier curves as well as the crude Kaplan-Meier curves were presented, and group comparisons were done using the adjusted log-rank test.

All statistical analyses were performed with SPSS software, version 24 (IBM Corp, Armonk, NY) and SAS version 9.4 (SAS Institute Inc, Cary, NC). A $P$ value of $<.05$ was considered statistically significant.

\section{RESULTS}

\section{Sarcopenia Defined From Psoas Muscle Area}

The distribution of psoas muscle area is shown in Figure 2, $C$. The average of psoas muscle area was $933 \pm 241 \mathrm{~mm}^{2}$ for men and $596 \pm 154 \mathrm{~mm}^{2}$ for women $(P<.001)$. Not surprisingly, psoas muscle area decreased with increasing age. In male patients, the area in those aged 80 years or older was smaller than that in patients aged between 70 and 79 years $\left(877 \pm 237 \mathrm{~mm}^{2}\right.$ vs $\left.953 \pm 240 \mathrm{~mm}^{2}, P=.044\right)$. In female patients, the difference in the area was not significant between those patients in their $80 \mathrm{~s}$ and $70 \mathrm{~s}\left(564 \pm 150 \mathrm{~mm}^{2}\right.$ vs $606 \pm 155 \mathrm{~mm}^{2}, P=.190$ ). Based on the definition for sarcopenia in this study, the cut-off value for sarcopenia was $770 \mathrm{~mm}^{2}$ in male and $495 \mathrm{~mm}^{2}$ in female patients. Thus, it was determined that there were 107 patients with sarcopenia and 321 patients without sarcopenia. 


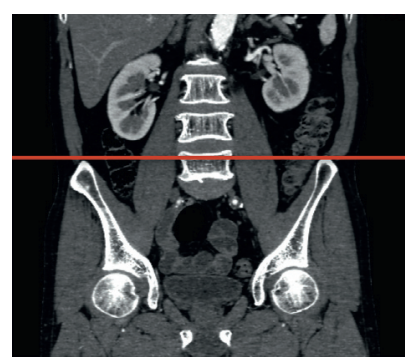

A

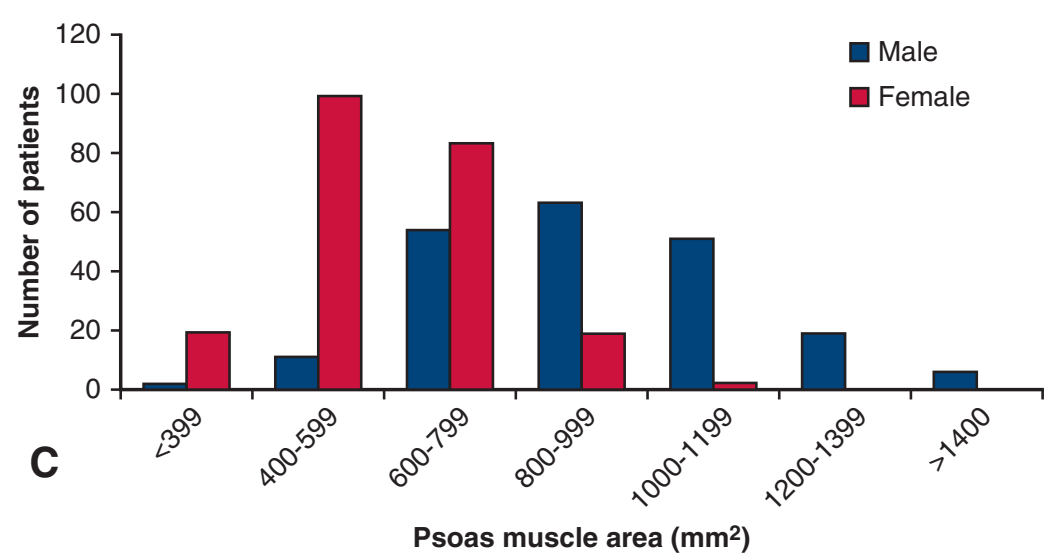

FIGURE 2. Measurement of psoas muscle area on preoperative computed tomography. A, Psoas muscle area was measured at the level of the top of the iliac crest (line). B, The psoas muscle borders were manually outlined. C, The distribution of psoas muscle area.

\section{Patient Characteristics in the Sarcopenia and Nonsarcopenia Groups}

Preoperative patient characteristics and operative data are shown in Table 1. Patients in the sarcopenia group were more likely than patients in the nonsarcopenia group to have smaller body surface area; lower BMI; a greater prevalence of New York Heart Association class 3/4; and certain comorbidities including chronic kidney disease, hemodialysis, and chronic obstructive pulmonary disease. Serum hemoglobin and albumin was significantly lower in the sarcopenia group than in the nonsarcopenia group. As for the relationship between sarcopenia and BMI, the prevalence of sarcopenia was $57 \%$ (41/72) in the underweight group (BMI $\left.<18.5 \mathrm{~kg} / \mathrm{m}^{2}\right), 19 \%(66 / 347)$ in the normal group (BMI between 18.5 and $30.0 \mathrm{~kg} / \mathrm{m}^{2}$ ), and $0 \%(0 / 9)$ in the obese group $\left(\mathrm{BMI}>30.0 \mathrm{~kg} / \mathrm{m}^{2}\right)$. Regarding type of surgical procedure, mitral valve replacement was performed more frequently in the sarcopenia group, as shown in Table 2.

\section{Clinical Outcomes}

Early outcomes are presented in Table 3. In the overall analysis, in-hospital mortality did not differ between the groups. The sarcopenia group had greater rates than the nonsarcopenia group did for stroke, intra-aortic balloon pump/percutaneous cardiopulmonary support use, and low cardiac output syndrome $(P=.012, P=.037$, and $P=.022$, respectively). Hemodialysis was newly induced in 8 patients $(1.9 \%)$ postoperatively; however, all patients were able to wean from hemodialysis at discharge.

Among the 413 survivors, late death occurred in 36 patients $(8.7 \%)$. The survival and freedom from MACCE results are shown in Figure 3, $A$ and $B$. The Kaplan-Meier actuarial survival rate at 5 years was $72.0 \pm 5.1 \%$ for the sarcopenia group and $91.1 \pm 1.8 \%$ for the nonsarcopenia group $(P<.001$; Figure 3, A). MACCE occurred in 24 patients $(5.8 \%)$ at follow-up; it included heart failure (10 patients), acute myocardial infarction (5 patients), reoperation for paravalvular leakage ( 3 patients), angina ( 2 patients), ventricular fibrillation ( 2 patients), stroke (1 patient), and unexplained death (1 patient). Freedom from MACCE at 5 years was $87.9 \pm 3.5 \%$ for the sarcopenia group and $94.3 \pm 1.7 \%$ for the nonsarcopenia group, respectively $(P=.005$; Figure 3 , $B)$. As for the impact of sarcopenia and sex, sarcopenia was shown to have a negative impact on late mortality in both sexes; however, sarcopenia negatively influenced freedom from MACCE only in male, not in female, patients (Figure E1). These findings suggest that the negative impact of sarcopenia is greater in males than in females.

\section{Predictors of Long-Term Survival and Freedom From MACCE}

To assess the impact of sarcopenia on the long-term outcomes, we performed Cox proportional hazards model for survival and freedom from MACCE (Table 4). On 
TABLE 2. Intraoperative data per study group

\begin{tabular}{|c|c|c|c|c|}
\hline Variable & Total $(n=428)$ & Sarcopenia $(n=107)$ & Nonsarcopenia $(n=321)$ & $P$ value \\
\hline AVR & $322(75.2 \%)$ & $73(68.2 \%)$ & $249(77.6 \%)$ & .070 \\
\hline Bioprosthesis & $287(89.1 \%)$ & $67(62.6 \%)$ & $220(68.5 \%)$ & .259 \\
\hline Mechanical valve & $35(10.9 \%)$ & $6(5.6 \%)$ & $29(9.0 \%)$ & .263 \\
\hline MVR & $95(22.2 \%)$ & $33(30.8 \%)$ & $62(19.3 \%)$ & .016 \\
\hline Bioprosthesis & $64(67.3 \%)$ & $26(24.3 \%)$ & $38(11.8 \%)$ & .002 \\
\hline Mechanical valve & $31(32.6 \%)$ & $7(6.5 \%)$ & $24(7.5 \%)$ & .747 \\
\hline MVP & $93(21.7 \%)$ & $28(26.2 \%)$ & $65(20.2 \%)$ & .223 \\
\hline Only ring annuloplasty & $49(52.7 \%)$ & $13(12.1 \%)$ & $36(11.2 \%)$ & .793 \\
\hline Resection and suture & $22(23.7 \%)$ & $6(5.6 \%)$ & $16(5.0 \%)$ & .800 \\
\hline Artificial chordae & $11(11.8 \%)$ & $6(5.6 \%)$ & $5(1.6 \%)$ & .022 \\
\hline Others & $11(11.8 \%)$ & $3(2.8 \%)$ & $8(2.5 \%)$ & .547 \\
\hline TAP & $174(40.7 \%)$ & $49(45.8 \%)$ & $125(38.9 \%)$ & .214 \\
\hline Maze procedure & $40(9.3 \%)$ & $8(7.5 \%)$ & $32(10.0 \%)$ & .566 \\
\hline Coronary artery bypass grafting & $88(20.6 \%)$ & $21(19.6 \%)$ & $67(20.9 \%)$ & .890 \\
\hline Combined procedures & $288(67.3 \%)$ & $75(70.1 \%)$ & $213(66.3 \%)$ & .475 \\
\hline Isolated valve surgery + nonvalvular procedure & $94(22.0 \%)$ & $17(15.9 \%)$ & $77(24.0 \%)$ & .082 \\
\hline AVR + MVR & $5(1.2 \%)$ & $3(2.8 \%)$ & $2(0.6 \%)$ & .102 \\
\hline AVR + MVP & $16(3.7 \%)$ & $6(5.6 \%)$ & $10(3.1 \%)$ & .246 \\
\hline AVR + TAP & $30(7.0 \%)$ & $5(4.7 \%)$ & $25(7.8 \%)$ & .382 \\
\hline $\mathrm{MVR}+\mathrm{TAP}$ & $42(9.8 \%)$ & $14(13.1 \%)$ & $28(8.7 \%)$ & .193 \\
\hline $\mathrm{MVP}+\mathrm{TAP}$ & $36(8.4 \%)$ & $12(11.2 \%)$ & $24(7.5 \%)$ & .232 \\
\hline $\mathrm{AVR}+\mathrm{MVR}+\mathrm{TAP}$ & $38(8.9 \%)$ & $12(11.2 \%)$ & $26(8.1 \%)$ & .330 \\
\hline $\mathrm{AVR}+\mathrm{MVP}+\mathrm{TAP}$ & $27(6.3 \%)$ & $6(5.6 \%)$ & $21(6.5 \%)$ & .822 \\
\hline $\mathrm{CPB}$ time, $\min$ & $167 \pm 49$ & $166 \pm 51$ & $167 \pm 49$ & .776 \\
\hline Crossclamp time, min & $137 \pm 41$ & $135 \pm 41$ & $138 \pm 41$ & .545 \\
\hline
\end{tabular}

Values for continuous variables are expressed as mean \pm standard deviation, and values for categorical variables are expressed as numbers (\%). AVR, Aortic valve replacement; $M V R$, mitral valve replacement; $M V P$, mitral valve repair; $T A P$, tricuspid annuloplasty; $C P B$, cardiopulmonary bypass.

multivariable analysis, sarcopenia was identified as an independent predictor for decreased survival and freedom from MACCE (hazard ratio, 2.221; 95\% confidence interval, $1.258-3.924 ; P=.006$ and hazard ratio, $2.802 ; 95 \%$ confidence interval, $1.242-6.319 ; P=.013$, respectively). Other predictors identified include BMI $>30.0 \mathrm{~kg} / \mathrm{m}^{2}$, chronic kidney disease, and serum albumin for late mortality. Diabetes was determined to be an independent risk factor for MACCE during follow-up. To circumvent the mismatch between limited number of events and high number of confounders, we performed inverse probability weighting using PS analysis (Figure 3, $C$ and $D$ ). The differences in the variables before and after PS weighting are shown in Table E1. After PS weighting, sarcopenia was associated

TABLE 3. In-hospital outcomes per study group

\begin{tabular}{|c|c|c|c|c|}
\hline Variable & Total $(n=428)$ & Sarcopenia $(n=107)$ & Nonsarcopenia $(n=321)$ & $P$ value \\
\hline In-hospital mortality & $15(3.5 \%)$ & $6(5.6 \%)$ & $9(2.8 \%)$ & .221 \\
\hline Stroke & $7(1.6 \%)$ & $5(4.7 \%)$ & $2(0.6 \%)$ & .012 \\
\hline IABP or PCPS use & $6(1.4 \%)$ & $4(3.7 \%)$ & $2(0.6 \%)$ & .037 \\
\hline Re-exploration for bleeding & $10(2.3 \%)$ & $4(3.7 \%)$ & $6(1.9 \%)$ & .463 \\
\hline New onset of atrial fibrillation & $143(33.4 \%)$ & $39(36.4 \%)$ & $104(32.4 \%)$ & .478 \\
\hline Prolonged ventilation use $>48 \mathrm{~h}$ & $37(8.6 \%)$ & $9(8.4 \%)$ & $28(8.7 \%)$ & 1.000 \\
\hline New need of hemodialysis & $8(1.9 \%)$ & $3(2.8 \%)$ & $5(1.6 \%)$ & .419 \\
\hline Low cardiac output syndrome & $13(3.0 \%)$ & $7(6.5 \%)$ & $6(1.9 \%)$ & .022 \\
\hline Permanent pacemaker implantation & $5(1.2 \%)$ & $2(1.9 \%)$ & $3(0.9 \%)$ & .603 \\
\hline Intensive care unit stay, $d$ & $3.2 \pm 3.1$ & $3.6 \pm 4.2$ & $3.1 \pm 2.6$ & .525 \\
\hline Discharge to health care facility & $54(12.6 \%)$ & $17(15.9 \%)$ & $37(11.5 \%)$ & .313 \\
\hline
\end{tabular}

Values for continuous variables are expressed as mean \pm standard deviation. Values for categorical variables are expressed as numbers (\%). IABP, Intra-aortic balloon pump; $P C P S$, percutaneous cardiopulmonary support. 


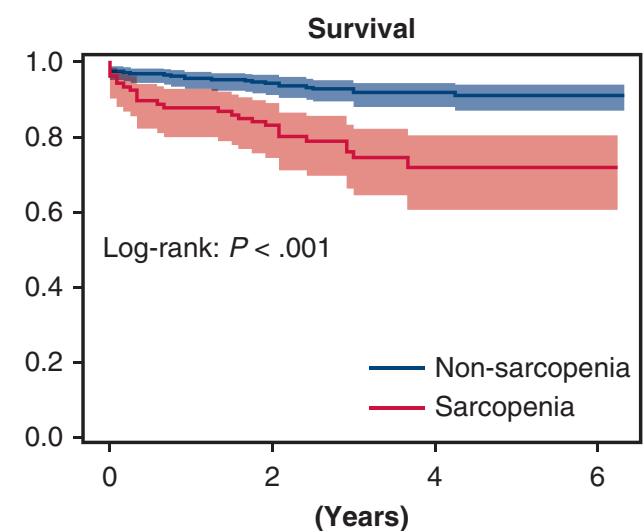

Number at risk Non-sarcopenia 321 Sarcopenia $\quad 107$

A

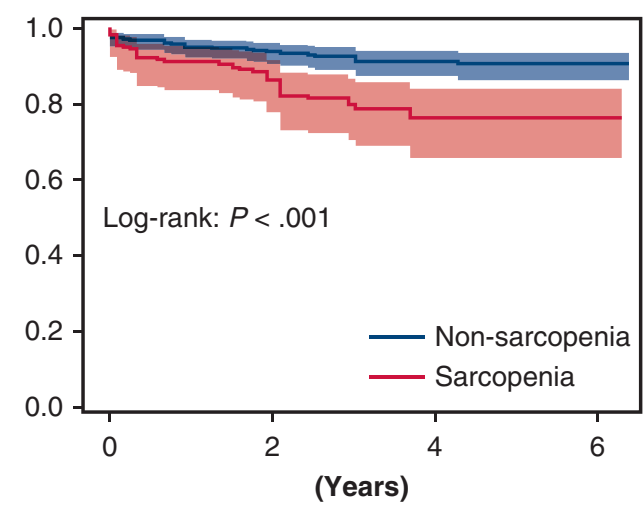

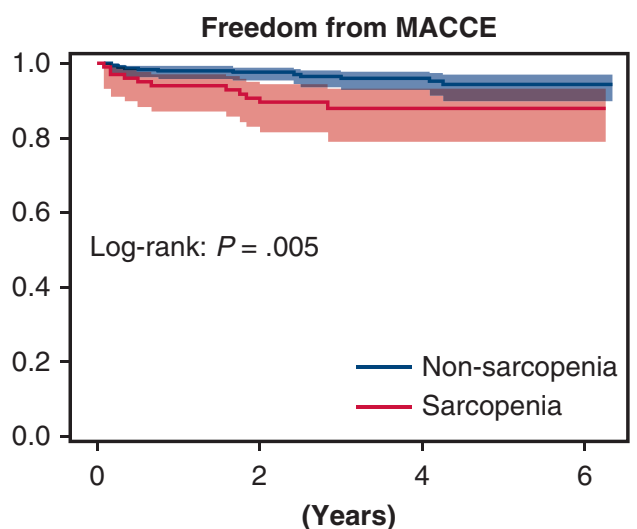

Number at risk Non-sarcopenia 321 Sarcopenia $\quad 107$ 282 124 19 20

B

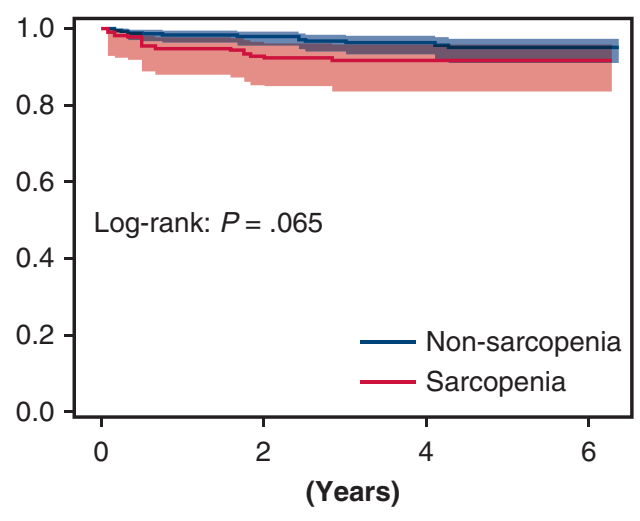

FIGURE 3. Kaplan-Meier curves of (A) overall survival and (B) freedom from MACCE with $95 \%$ confidence intervals before inverse propensity score weighing. Kaplan-Meier curves of (C) overall survival and (D) freedom from MACCE with $95 \%$ confidence intervals after inverse propensity score weighing. MACCE, Major adverse cardiac and cerebrovascular events.

with later mortality. As for freedom from MACCE, the difference did not reach significance between the sarcopenia and nonsarcopenia groups. The relationship between BMI and the hazard ratio for long-term outcomes is shown in Figure E2. For BMI $>30 \mathrm{~kg} / \mathrm{m}^{2}$, the hazard ratio for late mortality increased with increase in BMI.

\section{DISCUSSION}

The principal finding in this study was that preoperative sarcopenia derived from psoas muscle area resulted in a greater risk of long-term mortality and MACCE in elderly patients undergoing elective valve surgery. Frailty can be evaluated using different techniques, including the use of questionnaires and the assessment of certain physical activities. Frailty has been reported to outperform traditional anesthetic and surgical risk scores. ${ }^{17}$ The measurements of physical activities, including walking speed, can easily be influenced by comorbidities, cardiac function, and the timing to evaluate. Therefore, these measurements can be nonreproducible.
The term "sarcopenia" was proposed by Rosenberg ${ }^{18}$ to describe an age-related decrease of muscle mass in the late 1980s. The condition is characterized by age-associated generalized loss of skeletal muscle mass and muscle strength, ultimately resulting in an insidious functional decline. After age 50 years, muscle mass decreases at an annual rate of $1 \%$ to $2 \% .{ }^{19}$ There has been an increasing interest in the use of sarcopenia as an objective way to define frailty in the context of preoperative risk assessment. Sarcopenia, as assessed by total psoas volume, has been identified as an independent predictor of postoperative major complications, survival, and total hospital costs after surgery for abdominal malignancies. . $^{80-22}$

However, less is known about the impact of sarcopenia on the outcomes after cardiac surgery. Ganapathi and colleagues ${ }^{11}$ showed that a high frailty score was associated with discharge to another location other than home and 30-day and 1-year mortality in patients undergoing proximal aortic surgery. In their study, CT data for the measurement of psoas volume was, however, missing in 
TABLE 4. Cox proportional hazards analysis for independent risk factors for late mortality and MACCE

\begin{tabular}{|c|c|c|c|c|}
\hline \multirow[b]{2}{*}{ Variable } & \multicolumn{2}{|c|}{ Univariate } & \multicolumn{2}{|c|}{ Multivariable } \\
\hline & HR $(95 \%$ CI $)$ & $P$ value & HR $(95 \%$ CI) & $P$ value \\
\hline \multicolumn{5}{|l|}{ Late mortality } \\
\hline Age, y & $1.09(1.03-1.16)$ & .006 & $1.06(0.99-1.13)$ & .074 \\
\hline Sex (female) & $0.98(0.57-1.69)$ & .938 & - & \\
\hline Body surface area, $\mathrm{m}^{2}$ & $0.46(0.09-2.47)$ & .363 & - & \\
\hline Body mass index $<18.5 \mathrm{~kg} / \mathrm{m}^{2}$ & $1.27(0.64-2.54)$ & .493 & - & \\
\hline Body mass index $>30.0 \mathrm{~kg} / \mathrm{m}^{2}$ & $3.98(1.24-12.8)$ & .021 & $6.37(1.82-22.33)$ & .004 \\
\hline NYHA class $3 / 4$ & $2.76(1.58-4.85)$ & $<.0001$ & - & \\
\hline Hypertension & $0.91(0.50-1.65)$ & .755 & - & \\
\hline Diabetes & $1.35(0.73-2.50)$ & .337 & - & \\
\hline Ischemic heart disease & $1.68(0.94-3.01)$ & .082 & - & \\
\hline Atrial fibrillation & $1.35(0.76-2.38)$ & .305 & - & \\
\hline Cerebrovascular disease & $2.06(1.06-4.02)$ & .033 & $1.87(0.94-3.73)$ & .077 \\
\hline Chronic kidney disease* & $5.68(3.22-10.03)$ & $<.001$ & $4.72(2.37-9.40)$ & $<.001$ \\
\hline Hemodialysis & $3.60(1.54-8.45)$ & .003 & - & \\
\hline COPD & $3.66(1.32-10.16)$ & .013 & $2.26(0.76-6.77)$ & .144 \\
\hline Peripheral artery disease & $2.36(1.01-5.53)$ & .049 & - & \\
\hline Previous cardiac surgery & $1.76(0.55-5.65)$ & .344 & - & \\
\hline LVEF \% & $0.98(0.96-1.00)$ & .071 & - & \\
\hline Serum hemoglobin, $\mathrm{g} / \mathrm{dL}$ & $0.79(0.68-0.93)$ & .003 & - & \\
\hline Serum albumin, $g / d L$ & $0.17(0.11-0.27)$ & $<.001$ & $0.27(0.15-0.46)$ & $<.001$ \\
\hline Sarcopenia & $3.44(1.98-5.96)$ & $<.001$ & $2.47(1.34-4.55)$ & .004 \\
\hline \multicolumn{5}{|l|}{ Intraoperative data } \\
\hline AVR & $1.35(0.68-2.69)$ & .399 & - & \\
\hline MVR & $1.55(0.85-2.83)$ & .154 & - & \\
\hline MVP & $1.12(0.59-2.14)$ & .736 & - & \\
\hline TAP & $1.30(0.75-2.25)$ & .350 & - & \\
\hline Maze & $1.05(0.42-2.63)$ & .924 & - & \\
\hline CABG & $1.47(0.79-2.71)$ & .223 & - & \\
\hline Combined procedures & $0.97(0.54-1.74)$ & .927 & - & \\
\hline \multicolumn{5}{|l|}{ MACCE } \\
\hline Age, y & $1.06(0.96-1.16)$ & .245 & - & \\
\hline Sex (female) & $0.67(0.30-1.50)$ & .325 & - & \\
\hline Body surface area, $\mathrm{m}^{2}$ & $1.63(0.15-17.55)$ & 688 & - & \\
\hline Body mass index $<18.5 \mathrm{~kg} / \mathrm{m}^{2}$ & $0.77(0.23-2.57)$ & .664 & - & \\
\hline Body mass index $>30.0 \mathrm{~kg} / \mathrm{m}^{2}$ & $0.05(0.00-228543)$ & .700 & - & \\
\hline Body mass index, $\mathrm{kg} / \mathrm{m}^{2}$ & $1.03(0.92-1.15)$ & .630 & - & \\
\hline NYHA class $3 / 4$ & $2.54(1.13-5.73)$ & .024 & - & \\
\hline Hypertension & $0.57(0.25-1.29)$ & .178 & - & \\
\hline Diabetes & $2.58(1.15-5.82)$ & .022 & $2.46(1.06-5.73)$ & .037 \\
\hline Ischemic heart disease & $1.35(0.56-3.25)$ & .510 & - & \\
\hline Atrial fibrillation & $1.39(0.61-3.17)$ & .437 & - & \\
\hline Cerebrovascular disease & $1.09(0.33-3.65)$ & .891 & - & \\
\hline Chronic kidney disease* & $2.57(0.96-6.88)$ & .061 & - & \\
\hline Hemodialysis & $1.15(0.16-8.50)$ & .893 & - & \\
\hline COPD & $0.05(0.00-7772.66)$ & 620 & - & \\
\hline Peripheral artery disease & $3.63(1.24-10.62)$ & .019 & $2.74(0.89-8.46)$ & .080 \\
\hline Previous cardiac surgery & $0.05(0.00-956.21)$ & .546 & - & \\
\hline LVEF \% & $0.97(0.94-0.99)$ & .011 & $0.98(0.95-1.01)$ & .172 \\
\hline Serum hemoglobin, $\mathrm{g} / \mathrm{dL}$ & $1.00(0.80-1.26)$ & .992 & - & \\
\hline Serum albumin, $g / d L$ & $0.56(0.24-1.31)$ & .179 & - & \\
\hline Sarcopenia & $3.03(1.35-6.79)$ & .007 & $3.21(1.37-7.48)$ & .007 \\
\hline
\end{tabular}


TABLE 4. Continued

\begin{tabular}{llll}
\hline \multirow{2}{*}{ Variable } & \multicolumn{2}{c}{ Univariate } & Multivariable \\
\cline { 2 - 4 } & HR $(\mathbf{9 5} \% \mathbf{C I})$ & $\boldsymbol{P}$ value & HR (95\% CI) \\
\hline Intraoperative data & & & - \\
AVR & $0.54(0.24-1.23)$ & .140 & - \\
MVR & $2.77(1.23-6.23)$ & .014 & - \\
MVP & $1.84(0.79-4.30)$ & .160 & - \\
TAP & $2.97(1.27-6.94)$ & .012 & - \\
Maze & $2.55(0.95-6.82)$ & .063 & - \\
CABG & $1.27(0.50-3.19)$ & .617 & - \\
Combined procedures & $3.43(1.02-11.51)$ & .046 & - \\
\hline
\end{tabular}

HR, Hazard ratio; CI, confidence interval; NYHA, New York Heart Association; COPD, chronic obstructive pulmonary disease; $L V E F$, left ventricular ejection fraction; $A V R$, aortic valve replacement; $M V R$, mitral valve replacement; $M V P$, mitral valve repair; $T A P$, tricuspid annuloplasty; $C A B G$, coronary artery bypass grafting; $M A C C E$, major cardiac and cerebrovascular events. *Serum creatinine $>1.5 \mathrm{mg} / \mathrm{dL}$.

more than one half of the patients included. A couple of studies have reported the effects of sarcopenia on clinical outcomes after transcatheter treatment for structural heart disease; furthermore, in patients undergoing transcatheter aortic valve replacement, sarcopenia was specifically reported to be predictive of cumulative mortality. ${ }^{12,13}$

The definition of sarcopenia remains unestablished, and no definitive method and numerical values for parameters exist to define the condition. The European Working Group on Sarcopenia in Older People recommends cut-off points at 2 standard deviations below the mean reference value in healthy young adults. ${ }^{23}$ Most investigators have, however, used the lowest quartile of muscle area or volume as the definition of sarcopenia, and in this study we followed the same convention. ${ }^{8,9,11-14}$ Various options for measurement of muscle area or mass have been also suggested. ${ }^{11-13}$ Amini and colleagues ${ }^{9}$ demonstrated that the assessment of psoas muscle volume was a better means to predict postoperative complications than the measurement of psoas muscle area using a single axial cross-sectional image. However, volumetric analysis requires particular software and can be time-consuming.

Regarding the level of psoas muscle area measurement, some authors have measured the area at the level of the L3 or L4 vertebra. ${ }^{9,12,14,20}$ In this study, we measured the psoas muscle area at the level of the top of the iliac crest, which is commonly stated to cross the $\mathrm{L} 4,{ }^{24}$ because of several reasons. First, we considered the possibility of an anomaly of the vertebrae. In patients with an anomaly of the vertebrae, an exact measurement level can be hard to determine. Second, in elderly patients, compression fractures of the vertebrae, which can affect the level of measurement, is relatively common.

Unexpectedly, the rate of discharge to locations other than home was similar between the groups of patients with and without sarcopenia. This finding implies that postoperative rehabilitation may improve physical performance and help discharge patients to home, even in the cases of patients with sarcopenia.

Among 20 late deaths in the sarcopenia group, 8 patients preoperatively had chronic kidney disease and/or had undergone hemodialysis. This indicated that renal failure in the presence of sarcopenia was increasingly associated with late mortality after heart valve surgery.

There are several benefits of preoperative identification of sarcopenia. Sarcopenia is expected to predict late outcomes, which previous risk models have failed to capture. Integrating sarcopenia into risk assessment may better capture a patient's ability to tolerate surgery and can help steer therapy toward surgical, transcatheter, or medical treatment. Patients with sarcopenia may also benefit from interventions such as exercise training and nutritional supplementation preoperatively. Aggressive prehabilitation is expected to result in early mobilization after surgery, less frequency of morbidity related to immobilization, and further improvement of activity across the rest of the life course. Furthermore, the concept of sarcopenia as negative predictor of postsurgical mortality can also be applied to noncardiac surgery such as general surgery and thoracic surgery. $8,9,25$

\section{Study Limitations}

Several limitations of this study should be considered. First, this is a single-center study with a small sample size. Second, the cut-off value for sarcopenia was defined as the lowest quartile in this study, similar to that in previous studies. No universally accepted definition of sarcopenia currently exists, and further study is needed to determine the most accurate cut-off values for sarcopenia. Third, psoas muscle area measurement represents only one of the multiple dimensions of sarcopenia, which encompasses muscle mass, muscle strength, and physical performance. As such, combining the assessment of muscle mass and function might improve the prediction of the patient outcomes. Forth, most suitable cut-off values for sarcopenia potentially differ between races, sexes, and age groups. There are many different cut-off values used for the definition of sarcopenia in the existing literature. Thus, it is difficult to define a universal definition of sarcopenia. However, to maintain relative consistency with the previous literature, the cut-off values of total psoas area indexed to height in our cohort were 446 and $601 \mathrm{~mm}^{2} / \mathrm{m}^{2}$ for women and men, respectively. In each 


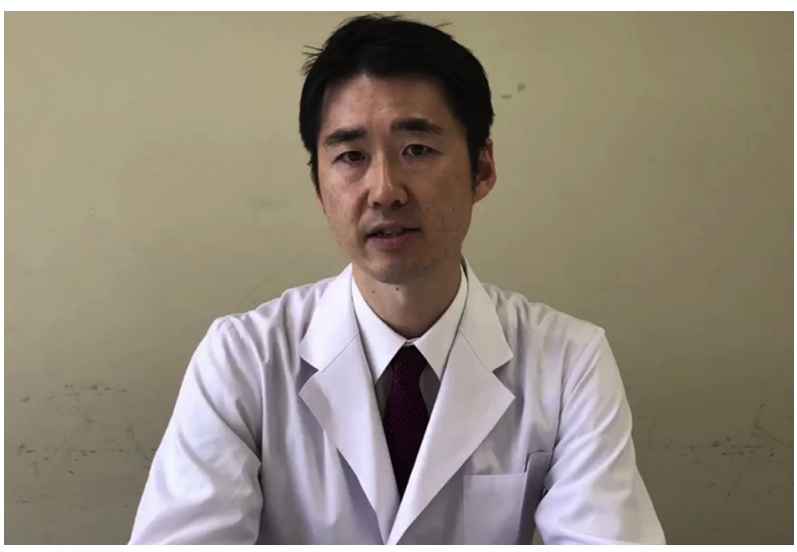

VIDEO 1. The corresponding author speaks about the importance and relevance of this study. Video available at: https://www.jtcvs.org/article/ S0022-5223(18)32032-4/fulltext.

population group, psoas muscle measurement in a certain number of patients or healthy people might be needed to more accurately define sarcopenia. Fifth, in this study, patients younger than 70 years old were excluded. Therefore, our cut-off value for sarcopenia cannot be applied for patients of all ages; however, frailty is mainly a concern in elderly patients, and including only elderly patients in a study can help identify true sarcopenic patients. Finally, 121 patients were excluded due to a lack of a preoperative abdominal CT scan, which could be a source for selection bias. As shown in Table E2, the 121 patients excluded were younger and had larger body surface area than the 427 patients included in the study. There were no difference in comorbidities.

\section{CONCLUSIONS}

Preoperative sarcopenia defined from the psoas muscle area was associated with long-term survival after valve surgery. Preoperative measurement of the psoas muscle area on CT scanning can be a useful tool for predicting long-term outcomes after valve surgery and objectively identifying frail patients before surgery (Video 1).

\section{Conflict of Interest Statement}

Authors have nothing to disclose with regard to commercial support.

\section{References}

1. Kieser TM, Rose MS, Head SJ. Comparison of logistic EuroSCORE and EuroSCORE II in predicting operative mortality of 1125 total arterial operations. Eur J Cardiothorac Surg. 2016;50:509-18.

2. Di Dedda U, Pelissero G, Agnelli B, De Vincentiis C, Castelvecchio S, Ranucci M. Accuracy, calibration, and clinical performance of the new EuroSCORE II risk stratification system. Eur J Cardiothorac Surg. 2013;43: 27-32.

3. Kuwaki K, Inaba H, Yamamoto T, Dohi S, Matsumura T, Morita T, et al. Performance of the EuroSCORE II and the Society of Thoracic Surgeons score in patients undergoing aortic valve replacement for aortic stenosis. J Cardiovasc Surg. 2015;56:455-62.
4. Xue QL. The frailty syndrome: definition and natural history. Clin Geriatr Med. 2011;27:1-15.

5. Afilalo J, Kim S, O'Brien S, Brennan JM, Edwards FH, Mack MJ, et al. Gait speed and operative mortality in older adults following cardiac surgery. JAMA Cardiol. 2016;1:314-21.

6. Lytwyn J, Stammers AN, Kehler DS, Jung P, Alexander B, Hiebert BM, et al. The impact of frailty on functional survival in patient 1 year after cardiac surgery. J Thorac Cardiovasc Surg. 2017;154:1990-9.

7. Morley JE, Anker SD, von Haehling S. Prevalence, incidence, and clinical impact of sarcopenia: facts, numbers, and epidemiology-update 2014. J Cachexia Sarcopenia Muscle. 2014;5:253-9.

8. Peng P, Hyder O, Firoozmand A, Kneuertz P, Schulick RD, Huang D, et al. Impact of sarcopenia on outcomes following resection of pancreatic adenocarcinoma. J Gastrointest Surg. 2012;16:1478-86.

9. Amini N, Spolverato G, Gupta R, Margonis GA, Kim Y, Wagner D, et al. Impact total psoas volume on short- and long-term outcomes in patients undergoing curative resection for pancreatic adenocarcinoma: a new tool to assess sarcopenia. J Gastrointest Surg. 2015;19:1593-602.

10. Nishimura RA, Otto CM, Bonow RO, Carabello BA, Erwin JP III, Guyton RA et al. 2014 AHA/ACC guideline for the management of patients with valvular heart disease: a report of the American College of Cardiology/American Heart Association task force on practice guidelines. J Am Coll Cardiol. 2014;63:e57-185.

11. Ganapathi AM, Englum BR, Hanna JM, Schechter MA, Gaca JG, Hurwitz KM, et al. Frailty and risk in proximal aortic surgery. J Thorac Cardiovasc Surg. 2014 147:186-91.

12. Mok M, Allende R, Leipsic J, Altisent OA, Del Trigo M, Campelo-Parada F, et al Prognostic value of fat mass and skeletal muscle mass determined by computed tomography in patients who underwent transcatheter aortic valve implantation. Am J Cardiol. 2016;117:828-33.

13. Paknikar R, Friedman J, Cron D, Deeb GM, Chetcuti S, Grossman PM, et al Psoas muscle size as a frailty measure for open and transcatheter aortic valve replacement. J Thorac Cardiovasc Surg. 2016;151:745-50.

14. Fujikawa H, Araki T, Okita Y, Kondo S, Kawamura M, Hiro J, et al. Impact of sarcopenia on surgical site infection after restorative proctocolectomy for ulcerative colitis. Surg Today. 2017;47:92-8.

15. Sabaté S, Mases A, Guilera N, Canet J, Castillo J, Orrego C, et al. Incidence and predictors of major perioperative adverse cardiac and cerebrovascular events in non-cardiac surgery. Br J Anaesth. 2011;107:879-90.

16. Austin PC. The use of propensity score methods with survival or time-to-event outcomes: reporting measures of effect similar to those used in randomized experiments. Stat Med. 2014;33:1242-58.

17. Keevil VL, Romero-Ortuno R. Ageing well: a review of sarcopenia and frailty. Proc Nutr Soc. 2015;74:337-47.

18. Rosenberg I. Summary comments: epidemiological and methodological problems in determining nutritional status of older persons. Am J Clin Nutr. 1989;50:1231-3.

19. von Haehling S, Morley JE, Anker SD. An overview of sarcopenia: facts and numbers on prevalence and clinical impact. J Cachexia Sarcopenia Muscle. 2010;1:129-33.

20. Valero V III, Amini N, Spolverato G, Weiss MJ, Hirose K, Dagher NN, et al Sarcopenia adversely impacts postoperative complications following resection or transplantation in patients with primary liver tumors. J Gastrointest Surg. 2015;19:272-81.

21. Jones KI, Doleman B, Scott S, Lund JN, Williams JP. Simple psoas cross-sectional area measurement is a quick and easy method to assess sarcopenia and predicts major surgical complications. Colorectal Dis. 2015;17:O20-6.

22. Gani F, Buettner S, Margonis GA, Sasaki K, Wagner D, Kim Y, et al. Sarcopenia predicts costs among patients undergoing major abdominal operations. Surgery. 2016;160:1162-71.

23. Cruz-Jentoft AJ, Baeyens JP, Bauer JM, Boirie Y, Cederholm T, Landi F, et al. Sarcopenia: European consensus on definition and diagnosis: report of the European working group on sarcopenia in older people. Age Ageing. 2010;39:412-23.

24. Chakraverty R, Pynsent P, Isaacs K. Which spinal levels are identified by palpation of the iliac crests and the posterior superior iliac spines. J Anat. 2007;210:232-6.

25. Suzuki Y, Okamoto T, Fujishita T, Katsura M, Akamine T, Takamori S, et al. Clinical implications of sarcopenia in patients undergoing complete resection for early non-small lung cancer. Lung Cancer. 2016;101:92-7.

Key Words: sarcopenia, frailty, heart valve surgery, psoas muscle area, risk assessment 
Survival

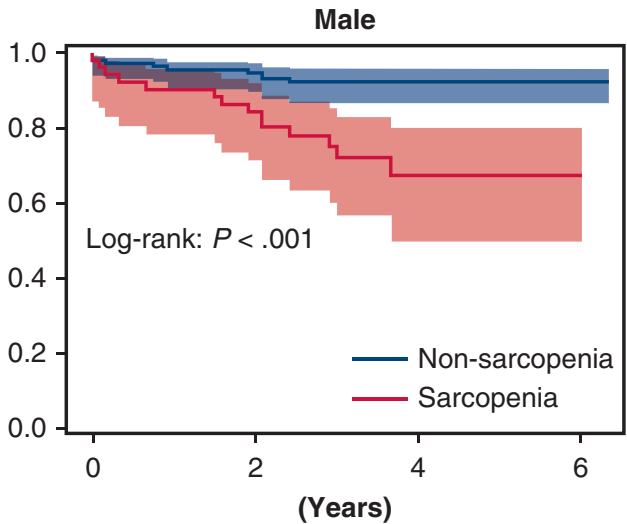

Number at risk

Non-sarcopenia 154

Sarcopenia

52

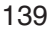

41

A

Freedom from MACCE

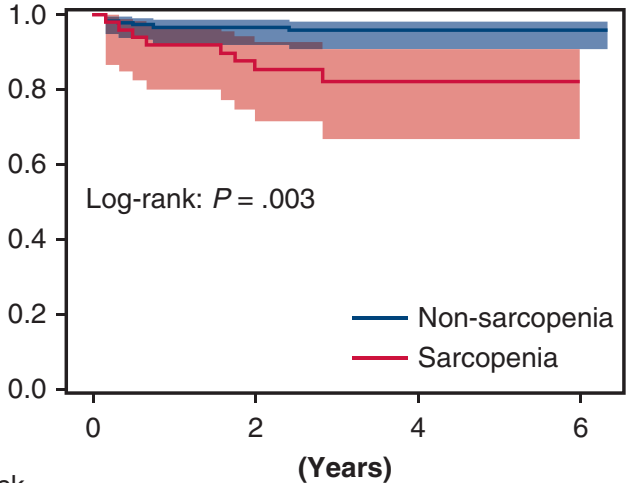

Number at risk

Non-sarcopenia 154

Sarcopenia

52

135

38

62
10

9

C

FIGURE E1. Kaplan-Meier curves of (A and B) overall survival and (C and D) freedom from MACCE stratified by sex with $95 \%$ confidence intervals. $M A C C E$, Major adverse cardiac and cerebrovascular events.

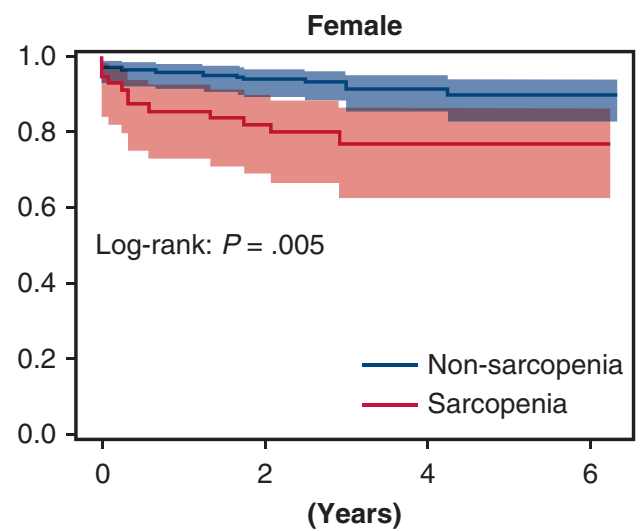

$\begin{array}{cccc}167 & 147 & 63 & 11 \\ 55 & 43 & 9 & 1\end{array}$

B

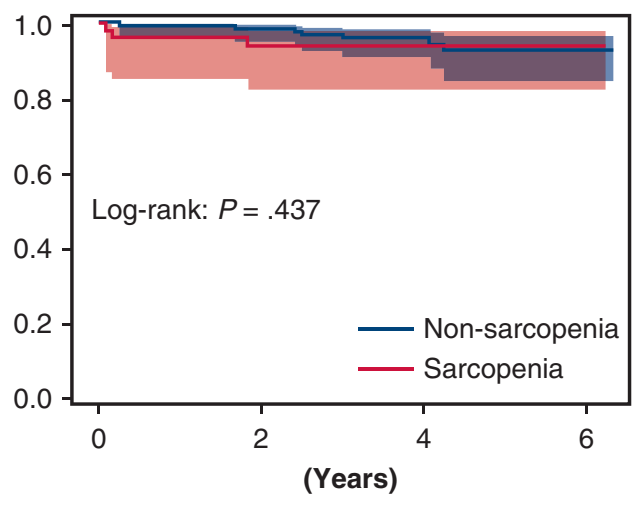

$\begin{array}{cccc}167 & 147 & 62 & 11 \\ 55 & 40 & 9 & 1\end{array}$

D
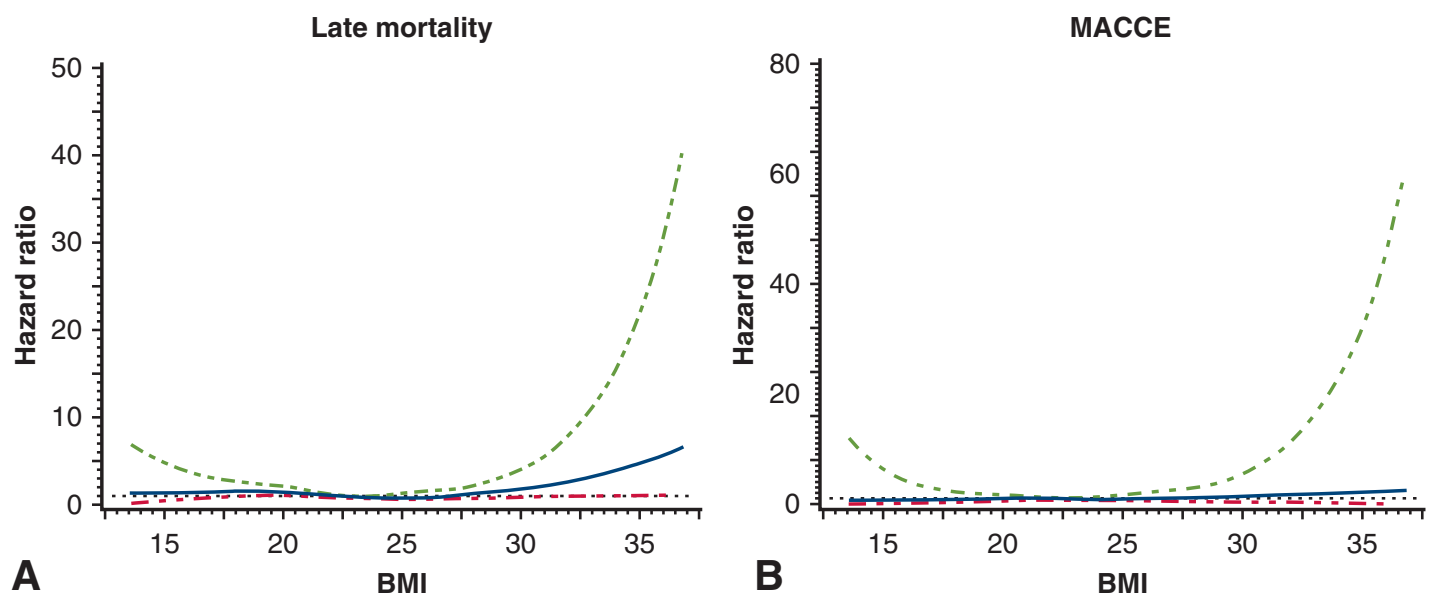

FIGURE E2. Hazard ratio for (A) overall survival and (B) MACCE based on body mass index with 95\% confidence intervals (dotted lines). $M A C C E$, Major adverse cardiac and cerebrovascular events; BMI, body mass index. 
TABLE E1. Differences in covariates at baseline and after applying IPW

\begin{tabular}{|c|c|c|c|c|c|c|}
\hline \multirow[b]{2}{*}{ Variable } & \multicolumn{3}{|c|}{ Baseline variables } & \multicolumn{3}{|c|}{ After IPW } \\
\hline & $\begin{array}{c}\text { Sarcopenia } \\
(n=107)\end{array}$ & $\begin{array}{c}\text { Nonsarcopenia } \\
(\mathbf{n}=\mathbf{3 2 1})\end{array}$ & SMD & $\begin{array}{c}\text { Sarcopenia } \\
(\mathbf{n}=107)\end{array}$ & $\begin{array}{c}\text { Nonsarcopenia } \\
(\mathbf{n}=\mathbf{3 2 0})\end{array}$ & SMD \\
\hline Age, $y$ & $77.0 \pm 4.6$ & $76.0 \pm 4.3$ & 0.217 & $75.9 \pm 4.0$ & $76.1 \pm 4.3$ & -0.041 \\
\hline Sex (female) & $55(51.4 \%)$ & $167(52.0 \%)$ & -0.012 & $(62.2 \%)$ & $(52.9 \%)$ & 0.189 \\
\hline Body surface area, $\mathrm{m}^{2}$ & $1.40 \pm 0.13$ & $1.52 \pm 0.16$ & -0.843 & $1.45 \pm 0.11$ & $1.48 \pm 0.17$ & -0.278 \\
\hline Body mass index, $\mathrm{kg} / \mathrm{m}^{2}$ & $19.6 \pm 2.8$ & $22.6 \pm 3.5$ & -0.953 & $21.4 \pm 3.1$ & $21.9 \pm 3.6$ & -0.149 \\
\hline $\begin{array}{l}\text { Body mass index category } \\
<18.5,18.5-30.0,>30.0\end{array}$ & $38.3 \%, 61.7 \%, 0 \%$ & $9.7 \%, 87.5 \%, 2.8 \%$ & -0.743 & $21.3 \%, 78.7 \%, 0 \%$ & $15.7 \%, 82.1 \%, 2.2 \%$ & -0.193 \\
\hline NYHA class $3 / 4$ & $51(47.7 \%)$ & $109(34.0 \%)$ & 0.282 & $(35.3 \%)$ & $(38.3 \%)$ & -0.066 \\
\hline Hypertension & $71(66.4 \%)$ & $231(72.0 \%)$ & -0.122 & $(71.1 \%)$ & $(71.3 \%)$ & -0.004 \\
\hline Diabetes & $23(21.5 \%)$ & $72(22.4 \%)$ & -0.023 & $(15.6 \%)$ & $(22.9 \%)$ & -0.186 \\
\hline Ischemic heart disease & $27(25.2 \%)$ & $70(21.8 \%)$ & 0.081 & $(19.4 \%)$ & $(25.3 \%)$ & -0.142 \\
\hline Atrial fibrillation & $36(33.6 \%)$ & $98(30.5 \%)$ & 0.067 & $(32.4 \%)$ & $(30.3 \%)$ & 0.046 \\
\hline Cerebrovascular disease & $14(13.1 \%)$ & $39(12.1 \%)$ & 0.028 & $(11.7 \%)$ & $(11.2 \%)$ & 0.016 \\
\hline Chronic kidney disease* & $19(17.8 \%)$ & $28(8.7 \%)$ & 0.269 & $(10.5 \%)$ & $(11.4 \%)$ & -0.029 \\
\hline Hemodialysis & $10(9.3 \%)$ & $7(2.2 \%)$ & 0.311 & $(4.0 \%)$ & $(4.8 \%)$ & -0.042 \\
\hline COPD & $7(6.5 \%)$ & $5(1.6 \%)$ & 0.255 & $(2.5 \%)$ & $(9.1 \%)$ & -0.020 \\
\hline Peripheral artery disease & $9(8.4 \%)$ & $16(5.0 \%)$ & 0.137 & $(5.9 \%)$ & $(7.0 \%)$ & -0.043 \\
\hline Previous cardiac surgery & $5(4.7 \%)$ & $12(3.7 \%)$ & 0.047 & $(4.0 \%)$ & $(4.3 \%)$ & -0.014 \\
\hline LVEF \% & $59.2 \pm 12.6$ & $61.3 \pm 12.4$ & -0.172 & $60.4 \pm 12.0$ & $60.5 \pm 13.0$ & -0.010 \\
\hline Serum hemoglobin, $\mathrm{g} / \mathrm{dL}$ & $11.5 \pm 1.6$ & $12.2 \pm 1.7$ & -0.440 & $11.8 \pm 1.4$ & $12.0 \pm 1.9$ & -0.144 \\
\hline Serum albumin, $\mathrm{g} / \mathrm{dL}$ & $3.9 \pm 0.5$ & $4.1 \pm 0.4$ & -0.500 & $4.0 \pm 0.5$ & $4.0 \pm 0.5$ & -0.066 \\
\hline AVR & $73(68.2 \%)$ & $249(77.6 \%)$ & -0.211 & $(76.9 \%)$ & $(75.8 \%)$ & 0.025 \\
\hline MVR & $33(30.8 \%)$ & $62(19.3 \%)$ & 0.268 & $(22.4 \%)$ & $(22.7 \%)$ & -0.005 \\
\hline MVP & $28(26.2 \%)$ & $65(20.2 \%)$ & 0.141 & $(19.7 \%)$ & $(22.3 \%)$ & -0.064 \\
\hline TAP & $49(45.8 \%)$ & $125(38.9 \%)$ & 0.139 & $(47.7 \%)$ & $(40.2 \%)$ & 0.152 \\
\hline Maze & $8(7.5 \%)$ & $32(10.0 \%)$ & -0.088 & $(13.4 \%)$ & $(9.3 \%)$ & 0.129 \\
\hline CABG & $21(19.6 \%)$ & $67(20.9 \%)$ & -0.031 & $(16.7 \%)$ & $(22.0 \%)$ & -0.135 \\
\hline Combined procedures & $75(70.1 \%)$ & $213(66.3 \%)$ & 0.080 & $(74.6 \%)$ & $(67.6 \%)$ & 0.155 \\
\hline
\end{tabular}

Values for continuous variables are expressed as mean \pm standard deviation, and values for categorical variables are expressed as numbers (\%). IPW, Inverse probability weighting; SMD, standardized mean difference; $N Y H A$, New York Heart Association; COPD, chronic obstructive pulmonary disease; $L V E F$, left ventricular ejection fraction; $A V R$, aortic valve replacement; $M V R$, mitral valve replacement; $M V P$, mitral valve repair; $T A P$, tricuspid annuloplasty; $C A B G$, coronary artery bypass grafting. *Serum creatinine $>1.5 \mathrm{mg} / \mathrm{dL}$. 
TABLE E2. Comparison of preoperative patient characteristics between the 428 patients included in this study and the 121 patients excluded due to a lack of preoperative computed tomography

\begin{tabular}{|c|c|c|c|}
\hline Variable & Patients included $(n=428)$ & Patients excluded $(n=121)$ & $P$ value \\
\hline Age, y & $76.3 \pm 4.4$ & $75.3 \pm 3.6$ & .010 \\
\hline Sex (female) & $222(51.9 \%)$ & $53(43.8 \%)$ & .123 \\
\hline Body surface area, $\mathrm{m}^{2}$ & $1.49 \pm 0.17$ & $1.53 \pm 0.17$ & .038 \\
\hline NYHA class $3 / 4$ & $160(37.4 \%)$ & $40(33.1 \%)$ & .395 \\
\hline Hypertension & $302(70.6 \%)$ & $87(71.9 \%)$ & .821 \\
\hline Diabetes & $95(22.2 \%)$ & $27(22.3 \%)$ & 1.000 \\
\hline Ischemic heart disease & $97(22.7 \%)$ & $24(19.8 \%)$ & .537 \\
\hline Atrial fibrillation & $134(31.3 \%)$ & $37(30.6 \%)$ & .912 \\
\hline Cerebrovascular disease & $53(12.4 \%)$ & $11(9.1 \%)$ & .422 \\
\hline Chronic kidney disease* & $47(11.0 \%)$ & $11(9.1 \%)$ & .619 \\
\hline Hemodialysis & $17(4.0 \%)$ & $5(4.1 \%)$ & 1.000 \\
\hline COPD & $12(2.8 \%)$ & $5(4.1 \%)$ & .550 \\
\hline Peripheral artery disease & $25(5.8 \%)$ & $2(1.7 \%)$ & .091 \\
\hline Previous cardiac surgery & $17(4.0 \%)$ & $5(4.1 \%)$ & 1.000 \\
\hline LVEF \% & $60.8 \pm 12.5$ & $61.6 \pm 10.9$ & .520 \\
\hline Serum hemoglobin, $g / d L$ & $12.1 \pm 1.7$ & $12.3 \pm 1.9$ & .148 \\
\hline Serum albumin, g/dL & $4.0 \pm 0.5$ & $4.1 \pm 0.4$ & .706 \\
\hline Psoas muscle area, $\mathrm{mm}^{2}$ & $758 \pm 262$ & NA & NA \\
\hline
\end{tabular}

Values for continuous variables are expressed as mean \pm standard deviation, and values for categorical variables are expressed as numbers (\%). NYHA, New York Heart Association; $C O P D$, chronic obstructive pulmonary disease; $L V E F$, left ventricular ejection fraction; $N A$, not available. *Serum creatinine $>1.5 \mathrm{mg} / \mathrm{dL}$. 